\title{
COVARIANT EXTENSION OF THE WIGNER TRANSFORMATION TO NON-ABELIAN YANG-MILLS SYMMETRIES FOR A VLASOV EQUATION APPROACH TO THE QUARK-GLUON PLASMA
}

\author{
J. Winter \\ Sektion Physik der Universität Mionchen, Theresienstr. 37, D-8000 Mïnchen 2, \\ F.R.G.
}

\begin{abstract}
Résumé - La transformation de Wigner est gênéralisêe comme une opération covariante par rapport à une symétrie de jauge locale. L'application à un champ de matière quantique de klein-Gordon en présence d'un champ classique self-consistent de Yang-Mills conduit à une extension correspondante de l'équation de Vlasov dans la limite classique et de ses corrections quantiques dont les principales corrections s'avèrent être des commutateurs caractéristiques dans les théories non-Abéliennes. Dans 1 'application à la chromodynamique on obtient un modèle semi-classique pour un plasma de quarks et gluons sans spin, ce qui devrait être utile au delà de la transition de dêconfinement.
\end{abstract}

\begin{abstract}
The Wigner transformation is generalized as a covariant operation with respect to a local gauge symmetry. The application to a quantum Klein-Gordon thatter-field in the presence of the non-quantized self-consistent Yang-Mills field leads to a corresponding extension of the Vlasov equation, in the classical limit, and moreover to its quantum corrections, the leading ones of which are found to be commutator terms, characteristic for non-Abelian theories. In the application to chromodynamics a semiclassical model for a plasma of spinless quarks and gluons is obtained, which should be of use beyond the deconfinement transition.
\end{abstract}

Since its introduction to describe the interaction of quarks in terms of a local gauge theory, quantum chromodynamics has not only been applied to the few-quark problem of elementary particle physics, but also to extended strongly interacting matter. Whereas current research is mainly concentrated on quark-gluon thermodynamics based on lattice QCD [1] to study in particular the deconfinement transition, for situations beyond this transition, at least, an alternative of a first approach might seem tempting which involves a simpler tool at the level of ordinary plasma physics: generalized Vlasov and Maxwell equations coupling the density matrix of quark matter and a classical mean gluon ("glue") field. The generalized Maxwell equation of such a concept would be just the non-quantized gluon field equation of QCD, with a classical quark-current calculated from the VIasov equation. The vlasov equation is established in the present work as the semi- 
classical limit of the quantum equation of motion for the quark two-point function, in the presence of the classical gluon field.

In combination with the wigner transformation of the equation of motion, such a jimit is well known as a convenient access to non-relativistic Vlasov equations from quantum mechanics [2]. In the following the concept of the wigner transform is generalized to relativistic non-Abelian local gauge theories, the structure including chromodynamics as well as, in the Abelian special case, electrodynamics, for which the standard vlasov equation is reproduced. To concentrate on essential treats in the argumentation, with neglection of spin the matter (quark) field is treated as a Klein-Gordon field.

The equation of motion for the two-point function or generalized density matrix, defined as the expectation value of space-time dependent Heisenberq field operators creating and annihilating a a spinless particle

$$
N_{c^{\prime} c^{\prime \prime}}^{\prime}\left(x^{\prime}, x^{\prime \prime}\right)=\left\langle\psi_{c^{\prime \prime}}^{\dagger}\left(x^{\prime \prime}\right) \psi_{c^{\prime}}\left(x^{\prime \prime}\right)\right\rangle \text {, }
$$

in the mean field (Hartree) approximation is obtained from the equation for the free klein-Gordon field by replacing ordinary derivatives by covariant derivatives $D$ implying the vector potential $A$ of a classical Yang-Mills field ${ }^{\dagger}$

$$
\begin{aligned}
& \left(D_{\mu}^{\prime} D^{\mu \prime}-m^{2}\right) N\left(x^{\prime}, x^{\prime \prime}\right)=0, \\
& D_{\mu}^{\prime} N\left(x^{\prime}, x^{\prime \prime}\right) \equiv \partial_{\mu}^{\prime} N+i A_{\mu}\left(x^{\prime}\right) N\left(x^{\prime}, x^{\prime \prime}\right) .
\end{aligned}
$$

The gauge field $\mathcal{A}$ and all other script-type quantities in this work are matrices in charge (colour) space, the charge indices being suppressed in the following. We use the standard conventions of $\hbar=1$ and of absorbing the coupling constant $g$ by $A$. The generalized vlasov equation will be derived from the essential anti-Hermitian part of the matrix equation

$$
\frac{1}{2 i}\left(D_{\mu}^{\prime} D^{\mu 1} N-N D_{\mu}^{\prime \prime} D^{\mu 1+}\right)=0
$$

the Hermitian part giving rise to a supplementary condition to its solution. The derivatives $N D_{\mu}^{\prime \prime}$ are defined in correspondence

\footnotetext{
'We choose the signature +++- of the metric
} 
with (3) with the convention of $d_{\mu}^{\prime \prime}$ acting to the left.

In the further transformation of eqs. (2) to the wigner representation in terms of midpoint $x$ and momentum $p$ it is essential to conserve the local gauge-invariant form. For the generalization of the standard wigner transformation of a chargescalax density matrix to the charge-tensor $\mathcal{N}(1)$ this principle means, that linear combinations of this quantity taken at different coordinate pairs $\left(x^{\prime}, x^{\prime \prime}\right)$ should result in a quantity which in local gauge transformations transforms as a local charge tensor at one definite point $x ; i$. e. the $N_{c^{\prime} c^{\prime \prime}}\left(x^{\prime}, x^{\prime \prime}\right)$ must be refered to the same local charge space at $x$ by parallel transfer to $x$ with regard to both charge indices. The wigner transform of $N$, or charge-tensor wigner function, has thus to be defined as

$$
\mathcal{N}(x, \rho)=\int d^{4} \xi e^{-i p_{\mu} \xi^{\mu}} U_{\ell}\left(x, x+\frac{1}{2} \xi\right) \mathcal{N}\left(x+\frac{1}{2} \xi, x-\frac{1}{2} \xi\right) U_{l}\left(x-\frac{1}{2} \xi, x\right)
$$

where $U$ is a unitary charge operator of finite parallel transfer [3]. The result of finite parallel transfer in general depends on the particular path chosen between the two points, the natural choice of which is the straight line, defining the matrix of "linear" parallel transfer $U_{\ell}$ used in (4), and which should be generalized to the geodesic for a possible extension of the theory to curved space.

on expressing $\mathcal{N}\left(x^{\prime}, x^{\prime \prime}\right)$ in the differential equation (2) in terms of $N(x, p)$ by means of the inverted wigner transformation, due to the unitarity of $U$ given by

$$
\begin{aligned}
& N\left(x^{\prime}, x^{\prime \prime}\right)=U_{\ell}\left(x^{\prime}, x\right) \tilde{N}(x ; \xi) U_{\ell}\left(x, x^{\prime \prime}\right), \\
& \tilde{N}(x ; \xi)=\int \frac{\alpha^{4} p}{(2 \pi)^{4}} e^{i p_{\mu} \xi^{\mu}} N(x, p), \quad x=\frac{1}{2}\left(x^{\prime}+x^{\prime \prime}\right), \xi=x^{\prime}-x^{\prime \prime},
\end{aligned}
$$

in order to end up with a kinetic equation for $N(x, p)$, the problem of permuting the operations of covariant differentiation and finite parallel transfer arises. For its treatment it is convenient, instead of directly calculating the second derivatives needed for (2), to evaluate them from the second covariant differentials 


$$
\begin{aligned}
& D^{\prime 2} \mathcal{N}\left(x^{\prime} x^{\prime \prime}\right)=D_{\mu}^{\prime} D_{\nu}^{\prime} \mathcal{N}\left(x^{\prime}, x^{\prime \prime}\right) d x^{\mu \prime} d x^{\nu \prime}, \\
& \mathcal{N}\left(x^{\prime}, x^{\prime \prime}\right) D^{\prime \prime+2}=\mathcal{N}\left(x_{1}^{\prime} x^{\prime \prime}\right) D_{\mu}^{\prime \prime+} D_{\nu}^{\prime \prime} d x^{\mu \prime \prime} d x^{\nu \prime \prime} .
\end{aligned}
$$

To begin with the first differentials for purposes of demonstration, the covariant change of expression (5a) for $\mathcal{N}\left(x^{\prime}, x^{\prime \prime}\right)$ due to a change $d x^{\prime}$ of its first argument is obtained as

$$
D^{\prime} \mathcal{N}\left(x_{1}^{\prime}, x^{n}\right)=\left(\Delta^{\prime} U_{l}^{\prime}\right) \tilde{N} U_{l}^{\prime \prime}+U_{l}^{\prime} \tilde{N}\left(\Delta^{\prime} U_{l}^{\prime \prime}\right)+u_{l}^{\prime}\left(D_{x}^{\prime} \tilde{N}\right) U_{l}^{\prime \prime}
$$

with $U_{l}^{\prime} \equiv U_{l}\left(x^{\prime}, x\right), U_{l}^{\prime \prime} \equiv U_{l}\left(x, x^{\prime \prime}\right)$. The quantities $\Delta U_{l}$ stand for a variation of the matrix of linear parallel transfer between two points $x_{1}$ and $x_{2}$, defined as a local covariant vector with regard to each of the two points and charge indices lcovariant bi-vector). For arbitrary changes of $x_{1}$ and $x_{2}$ this variation has the form

$$
\begin{aligned}
\Delta U_{\ell}\left(x_{2}, x_{1}\right) \equiv & U_{\ell}\left(x_{2}+d x_{2}, x_{1}+d x_{1}\right)-U_{\ell}\left(x_{2}, x_{1}\right) \\
& +i A_{\mu}\left(x_{2}\right) d x_{2}^{\mu} U_{l}\left(x_{2}, x_{1}\right)-i U_{l}\left(x_{2}, x_{1}\right) A_{\mu}\left(x_{1}\right) d x_{1}^{\mu} ;
\end{aligned}
$$

$\Delta^{\prime}$ in (7) means the specification of (8) to changes of the arguments $x_{1}=x=\frac{1}{2}\left(x^{\prime}+x^{\prime \prime}\right), x_{2}=x^{\prime}$ or $x_{1}=x^{\prime \prime}, x_{2}=x$ due 'a change $d x^{\prime}$ with $d x^{\prime \prime}=0$; Iikewise, for a corresponding calculation of $N D^{\prime \prime}, \Delta^{\prime \prime}$ is referred to changes $d x^{\prime \prime}$. As is easily verified, use of definition (8) in the first two terms of (7) goes along with a differential $D_{x}^{\prime} \tilde{N}$ in the third term, which combines the covariant derivative with regard to the midpoint $x$ with the usual dexivative with regard to the relative coordinate $\xi$

$$
D_{x}^{\prime} \tilde{N} \equiv\left(\frac{1}{2} D_{\mu}+\frac{\partial}{\partial \xi^{\mu}}\right) \tilde{N} d x^{\mu^{\prime}}
$$

in contrast to the covariant vector derivatives $D_{\mu}^{\prime}$ and $D_{\mu}^{\prime \prime}, D_{\mu}$ in (9) is the covariant derivative of a second-rank tensor

$$
D_{\mu} \tilde{N} \equiv \frac{\partial}{\partial x^{\mu}} \tilde{N}+i\left[A_{\mu}(x), \tilde{N}\right] .
$$


For the second differentials needed for the right side of eqs. (6), a calculation similar to the preceding one leads to the result

$$
\begin{aligned}
& D^{\prime 2} \mathcal{N}\left(x^{\prime}, x^{\prime \prime}\right)=\left(\Delta^{\prime 2} U_{l}^{\prime}\right) \tilde{N} U_{l}^{\prime \prime}+U_{l}^{\prime} \tilde{N}\left(\Delta^{\prime 2} U_{l}^{\prime \prime}\right)+U_{l}^{\prime}\left(D_{x}^{\prime 2} \tilde{N}\right) U_{\ell}^{\prime \prime} \\
& \quad+2\left(\left(\Delta^{\prime} U_{l}^{\prime}\right) \tilde{N}\left(\Delta^{\prime} U_{l}^{\prime \prime}\right)+\left(\Delta^{\prime} U_{i}^{\prime}\right)\left(D_{x}^{\prime} \tilde{N}\right) U_{l}^{\prime \prime}+U_{l}^{\prime}\left(D_{x}^{\prime} \tilde{N}\right)\left(\Delta^{\prime} U_{\ell}^{\prime \prime}\right)\right)
\end{aligned}
$$

here the second variation $\Delta^{2} U_{l}$ of the parallel transfer matrix is defined by substituting $\Delta U_{l}$ for $U_{4}$ in (8) and the corresponding conventions for $\Delta^{\prime 2}$ and $\Delta^{\prime \prime 2}$. The left side of $(6 \mathrm{~b})$ is likewise given by (11) with replacement of $\Delta^{\prime}$ and $D_{x}{ }^{\prime}$

$$
\begin{aligned}
& N\left(x^{\prime}, x^{\prime \prime}\right) D^{\prime \prime 2}=\left\{\Delta^{\prime} \rightarrow \Delta^{\prime \prime}, D_{x}^{\prime} \tilde{\mathcal{N}} \rightarrow D_{x}^{\prime \prime} \tilde{\mathcal{N}}\right\}, \\
& D_{x}^{\prime \prime} \tilde{N}=\left(\frac{1}{2} D_{\mu}-\frac{\partial}{\partial \xi^{\mu}}\right) \tilde{\mathcal{N}} d x^{\mu \prime \prime} .
\end{aligned}
$$

The variatious $\Delta U$ and $\Delta^{2} U$ of the matrix of finite parallel transfer between two points must be determined as the total effect of infinitesimal changes of all coordinates of the path connecting these points. In the following we first derive a general result for arbitrary variations of arbitrary connecting paths which is then specified to linear parallel transfer between two infinitesimally shifted end-points. - The treatment is based on the differential equation [3] for the parallel transfer matrix $U_{t}$ along a curve $4=t(s), 0 \leq s \leq 1, t(0)=x_{1}, t(1)=x_{2}$,

$$
i \frac{D}{d s} U_{t}(s) \equiv\left(i \frac{d}{d s}-A_{\mu}(t(s)) u^{\mu}(s)\right) U_{t}(s)=0, u^{\mu}=\frac{d t^{\mu}}{d s},
$$

which solved for the initial condition

$$
u_{t}(0)=y
$$

yields $U_{t}\left(x_{2}, x_{1}\right)$ at $s=1$. A formal explicit solution of (12) in terms of the Feynman path ordering operator [4] is feasible but not needed for the purpose of this work. Instead we have to investigate the variation of the solution due to infinitesimal 
variations $\delta t$ of the curve $t$ by means of Taylor expansion about its unvaried value. As in (12) the left point of $U\left(x_{2}, x_{1}\right)$ has been connected to the variable of the differential equation, the variations most directly accessible to calculation should be defined as covariant vectors with regard to the left point only,

$$
\begin{aligned}
& \delta U_{t}(s) \equiv U_{t+\delta t}-U_{t}+i A_{\mu}(t(s)) \delta t^{\mu}(s) U_{t}, \\
& \delta^{2} U_{t}(s)=\delta U_{t+\delta t}-\delta U_{t}+i A_{\mu}(t(s)) \delta t^{\mu}(s) \delta U_{t} .
\end{aligned}
$$

Insertion of $t(s)+\delta t(s)$ for $t(s)$ in eq. (12), consistent first-order expansion and subtraction of the zero-order equation immediately yields an equation for the ordinary variation

$$
i \frac{D}{d s}\left(U_{t+\delta t}-U_{t}\right)=\left(A_{\mu}(t) \frac{d\left(\delta t^{\mu}\right)}{d s}+\frac{\partial A_{\mu}}{\delta x^{\nu}} u^{\mu} \delta t^{\nu}\right) U_{t} .
$$

Applying the operator iD/ds to both sides of the definition (14) and using (16) and (12) one ends up with a linear-inhomogeneous differential equation for $\delta U$ which involves the fieldstrength tensor $\mathcal{F}$ in its source term

$$
\begin{aligned}
& i \frac{D}{d s} \delta U_{t}(s)=F_{\mu \nu}(t(s)) U_{t}(s) u^{\nu}(s) \delta t^{\mu}(s), \\
& F_{\mu \nu} \equiv \partial_{\mu} A_{\nu}-\delta_{\nu} A_{\mu}+i\left[A_{\mu}, A_{\nu}\right] ;
\end{aligned}
$$

a].] terms beyond the first in $F$ are contributed by the third term of (14), the first term of (16) being cancelled. Eq. (17) may be regarded as the Yang-Milis analogue of the equation for the geodesic deviation in gravitation theory which may be obtained by the same Taylor expansion technique [5] from the geodesic equation corresponding to eq. (12). The greater complexity of those equations lies in the fact that the varied curve (geodesic) there is not independently given but determined as solution of the equation itself together with its unit tangential vector $u$, which takes the place of the matrix $U$ of finite parallel transfer in (12) and (17). 
For obtaining a similar equation for the second variation $\delta^{2} U$ the same substitution $t \rightarrow t+\delta t$ and Taylor expansion are now performed in eq. (17) which, after cancelling of first-order quantities, yield as relation between second-order terms, including a second variation $\delta^{2} t$ of the curve

$$
\begin{aligned}
, \frac{D}{d s}\left(\delta U_{t+\delta t}-\delta U_{t}\right) & =\left(A_{\mu} \frac{d\left(\delta t^{\mu}\right)}{d s}+\frac{\partial A_{\mu}}{\partial x^{\nu}} u^{\mu} \delta t^{\nu}\right) \delta U_{t} \\
& +\left(\frac{\partial F_{\mu \nu}}{\partial x^{\lambda}} u^{\nu} \delta t^{\lambda} \delta t^{\mu}+\mathcal{F}_{\mu \nu} \frac{d\left(\delta t^{\nu}\right)}{d s} \delta t^{\mu}+\mathcal{F}_{\mu \nu} u^{\nu} \delta^{2} t^{\mu}\right) U_{t} \\
& +\tilde{F}_{\mu \nu}\left(U_{t+\delta t}-U_{t}\right) u^{\nu} \delta t^{\mu} ;
\end{aligned}
$$

the final differential equation obtained by applying $i D / d$ s to (15), inserting (19) and using again (12) and (17) for the last terms in (19) and (15) is

$$
\begin{aligned}
i \frac{D}{d s} \delta^{2} U= & 2 \mathcal{F}_{\mu \nu} u^{\nu} \delta t^{\mu} \delta U \\
& \left.+\mathcal{F}_{\mu \nu}\left(\delta u^{\nu} \delta t^{\mu}+u^{\nu} \delta^{2} t^{\mu}\right)+\left(D_{\lambda} \mathcal{F}_{\mu \nu}\right) u^{\nu} \delta t^{\mu} \delta t^{\lambda}\right) U,
\end{aligned}
$$

with the covariant tensor derivative defined by (10). The same result, through a lengthier calculation, is obtained by direct second-order Taylor expansion in (12) with the substitution $t \rightarrow t+\delta t+\frac{1}{2} \delta^{2} t$.

According to the definitions (14) and (15) of $\delta U$ and $\delta^{2} U$, equations (17) and (20) have to be solved with the initial conditions

$$
\delta U_{t}(0)=i A_{\mu}(0) \delta t^{\mu}(0), \quad \delta^{2} U_{t}(0)=-A_{\mu}(0) A_{\nu}(0) \delta t^{\mu}(0) \delta t^{\nu}(0) \quad(21 a, b)
$$

The explicit solutions of the linear-inhomogeneous differential equations are easily constructed by utilizing the matrix $U(t)$ for finaino both a particular solution as well as the general solution of the homogeneous equation to be adapted to (21). As turns out the solutions take their simplest form just if accomplished as covariant bi-vectors $\Delta U$ and $\Delta^{2} U$ in conformity 
with (8), which are ultimately needed in the application of this work,

$$
\begin{aligned}
\Delta U_{t}(s) & =\delta U_{t}(s)-i U_{t}(s) A_{\mu}(0) \delta t^{\mu}(0) \\
\Delta^{2} U_{t}(s) & =\delta^{2} U_{t}(s)-i \delta U_{t}(s) A_{\mu}(0) \delta t^{\mu}(0) \\
& =\delta^{2} U_{t}-2 i \delta U_{t} A_{\mu}(0) \delta t^{\mu}(0)+U_{t} A_{\mu}(0) A_{\nu}(0) \delta t^{\mu}(0) \delta t^{\nu}(0) .
\end{aligned}
$$

The explicit solutions are successively obtained as

$$
\begin{aligned}
& \Delta U_{t}(s)=-i U_{t}(s) \int_{0}^{s} d \sigma \tilde{F}_{\mu \nu}\left(\sigma^{\prime}\right) u^{\nu}(\sigma) \delta t^{\mu}(s) \\
& \delta^{2} U_{t}(s)=U_{t}(s)\left(-i \int_{0}^{s} d \sigma\left(i \frac{D}{d \sigma} \delta^{2} U_{t}(\sigma)\right)-A_{\mu}(0) A_{\nu}(0) \delta t^{\mu}(0) \delta t^{\nu}(0)\right)
\end{aligned}
$$

or, inserting the right side of (20) and using (23b) and (24)

$$
\begin{aligned}
& \Delta^{2} U_{t}(s)=-i U_{t}(s) \int_{0}^{s} d \sigma\left(\left(\tilde{F}_{\mu \nu}\left(\delta u^{\nu} t^{\mu}+u^{\nu} \delta^{2} t^{\mu}\right)+\left(\tilde{D}_{\lambda} \widetilde{F}_{\mu \nu}\right) u^{\nu} \delta t^{\mu} \delta t^{\lambda}\right)_{\varepsilon}\right. \\
& -2 i\left(\widetilde{F}_{\mu \nu} u^{\nu} \delta t^{\mu}\right)_{\epsilon} \int_{0}^{\sigma} d \bar{\sigma}\left(\tilde{F}_{\overline{\mu \nu}} u^{\bar{\nu}} \hat{\delta} t^{\bar{\mu}}\right)_{\bar{\sigma}} ;
\end{aligned}
$$

the abbreviation

$$
\tilde{F}_{\mu \nu}(s) \equiv U_{t}^{\dagger}(s) \mathcal{F}_{\mu \nu}(t(s)) U_{t}(s)
$$

together with a corresponaing definition of $\tilde{D} \tilde{f}$ is applied, and use of the unitarity of $U_{t}$ has been made.

The general result (24) and (25) now allows the calculation of the covariant differentials (11) and (11') and the following evaluation of the derivatives involved by the equation of motion (2). This is achieved through the following steps: 
i) the insertions

$$
\begin{aligned}
& t(s)=x_{1}+s\left(x_{2}-x_{1}\right), u=x_{2}-x_{1}, \\
& \delta t(s)=d x_{1}+s\left(d x_{2}-d x_{1}\right), \delta u=d x_{2}-d x_{1}, \\
& \delta^{2} t \equiv 0
\end{aligned}
$$

in (24) and (25) specify these relations for the linear parallel transfer yielding $\Delta u_{\ell}\left(x_{2}, x_{1}\right)$ and $\Delta^{2} U_{\ell}$ according to the definition (8);

ii) the specifications of $\Delta U_{\ell}$ and $\Delta^{2} U_{\ell}$ with regard to variation of the first or the second coordinate in the density matrix, as discussed after eq. (8) and to be inserted to (11) and (11'), are

$$
\begin{array}{ll}
\Delta^{\prime} U_{l}^{\prime}=\left.\Delta U_{\ell}\left(x^{\prime}, x\right)\right|_{d x=\frac{1}{2} d x^{\prime}} & , \Delta^{\prime \prime} U_{\ell}^{\prime}=\left.\Delta U_{\ell}\left(x^{\prime}, x\right)\right|_{d x=\frac{1}{2} d x^{\prime \prime}, d x^{\prime}=0}(28 a) \\
\Delta^{\prime} U_{l}^{\prime \prime}=\left.\Delta U_{\ell}^{+}\left(x^{\prime \prime}, x\right)\right|_{d x=\frac{1}{2} d x^{\prime}, d_{x}^{\prime \prime}=0}, \Delta^{\prime \prime} U_{\ell}^{\prime \prime}=\left.\Delta U_{\ell}^{+}\left(x^{\prime \prime}, x\right)\right|_{d x=\frac{1}{2} d x^{\prime \prime} \quad ; \quad(28 b)}
\end{array}
$$

in $(28 \mathrm{~b}), \Delta U_{\ell}\left(x, x^{\prime \prime}\right)$ is expressed by $\Delta u_{l}^{\dagger}\left(x^{\prime \prime}, x\right)$ in order to conveniently shift the midpoint $x$ to the right argument as the starting point $t(0)$ of the parallel transfer in all applications of $(24)$ - $(26)$, as well as to isolate a factor $u_{l}\left(x, x^{\prime \prime}\right)$ at the right of all terms in (11) and $\left(11^{\prime}\right)$, corresponding to a factor $U_{\ell}\left(x^{\prime}, x\right)$ at the left; $\Delta^{2} U$ is treated similarly; iii) inserting (28) to (11) and (11'), evaluating from these differentials the matrix of the second derivatives defined by (6), and forming the trace of this matrix, one finally obtains, after a retransformation of the factors $U_{l}$ to the left side of the relation,

$$
\begin{aligned}
& U_{\ell}\left(x, x^{\prime}\right)\left\{\begin{array}{l}
D_{\mu}^{\prime} D^{\mu \prime} N\left(x_{i}^{\prime} x^{\prime \prime}\right) \\
N\left(x^{\prime}, x^{\prime \prime}\right) D_{\mu}^{\prime \prime} D_{\mu}^{\prime \prime}
\end{array}\right\} U_{\ell}\left(x^{\prime \prime}, x^{\prime}\right)= \\
& \left(\frac{1}{2} D_{\mu} \pm \frac{\partial}{\partial \xi^{\mu}}\right)\left(\frac{1}{2} D^{\mu} \pm \frac{\partial}{\partial \xi}\right) \tilde{N}
\end{aligned}
$$




$$
\begin{aligned}
& +\frac{1}{i} \xi^{\nu} \int_{0}^{1} d s\left[\frac{1}{2}(1 \pm s) \tilde{F}_{\nu}^{\mu l}(s)\left(\left(\frac{1}{2} D_{\mu} \pm \frac{\partial}{\partial \xi^{\mu}}\right) \tilde{N}\right)+\frac{1}{2}(1 \mp s)\left(\left(\frac{1}{2} D_{\mu} \pm \frac{\partial}{\partial \xi^{\mu}}\right) \tilde{N}^{\prime}\right) \widetilde{F}_{\nu \mu}^{\mu}(s)\right. \\
& \left.+\frac{1}{8}(1 \pm s)^{2}\left(D^{\mu} \tilde{F}_{\mu \nu}\right)_{s}^{\prime} \tilde{N}+\frac{1}{8}(1 \mp s)^{2} \tilde{N}\left(D^{\mu} \tilde{F}_{\mu \nu}\right)_{s}^{\prime \prime}\right] \\
& -\frac{1}{8} \xi^{\nu} \xi^{\bar{\nu}} \iint_{0}^{1} d s d \bar{s}\left[(1 \pm s)(1 \mp \bar{s}) \tilde{F}_{\mu \nu}^{\prime}(s) \tilde{N}_{\tilde{F}}^{\tilde{F}^{\mu}} \bar{\nu}(\bar{s})\right.
\end{aligned}
$$

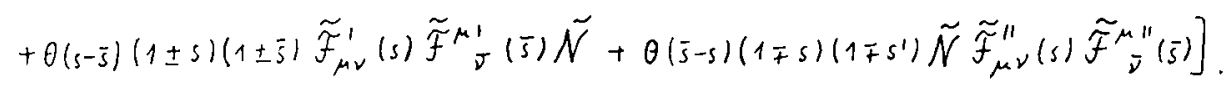

In (29) the effect of the finite parallel transfer has been completely absorbed by unitary transformations (26) of the field tensor or its derivative; by $\widetilde{F}^{\prime}$ and $\tilde{F}^{\prime \prime}$ we distinguish parallel transfer from $x$ to $x^{\prime}$ and to $x^{\prime \prime}$. A further formal absorption of $U$ by covariant derivatives is possible, if, assuming sufficient analytic properties of $\mathcal{F}$, expression $(26)$ for $\widetilde{F}(s)$ is replaced by its Taylor expansion about the midpoint $x$ at $s=0$, which due to (12), (27b) and (5d) gets a compact form involving the exponential of the covariant tensor derivative (10),

$$
\left\{\begin{array}{l}
\widetilde{F}_{\mu \nu}^{\prime}(s) \\
\widetilde{F}_{\mu \nu}^{\prime \prime}(s)
\end{array}\right\}=\left(\exp \pm \frac{1}{2} s \xi^{\lambda} D_{\lambda}\right) F_{\mu \nu}(x)
$$

With the insertion of (30) the integrations in (29) may be explicitly performed; subtraction of (29a) and (29b) and evaluation of the form $(5 c)$ for $\tilde{\mathcal{N}}$ by substituting $\xi^{\nu} \rightarrow i d / \partial p_{\nu}$, $i^{-1} \delta / d \xi^{\nu} \rightarrow p_{\nu}$ would then lead to a completely equivalent form of the equation of motion (2a) as a formal partial differential equation for the wigner transform $N(x, p)(4)$, where all orders of the differential operators $D_{\nu}$ and $\partial / \partial p_{\nu}$ are involved. The expansion (30) into terms involving increasing derivatives of the field-strength at the midpoint has the meaning of a semiclassical expansion of the quantum equations similar to the expansion of products of wigner transforms in the non-relativistic case [6], and can be truncated for sufficient homogeneity of the field.In the present work we restrict ourselves to first-order expansion of (30) including the gradient correction, and to expansion to the same order (in $\dot{n}$ ) of the equation of motion after insertion 
of (30). The result has the compact form

$$
\begin{aligned}
& p^{\mu} D_{\mu} N+\frac{1}{2} p^{\mu} \frac{\partial}{\partial p^{\nu}}\left\{\mathcal{F}_{\mu}^{\nu}, N\right\} \\
& -\frac{i}{8}\left(\frac{\partial}{\partial p^{\nu}}\left[\mathcal{F}^{\mu \nu}, D_{\mu} N\right]-p^{\mu} \frac{\partial}{\partial p^{\nu}} \frac{\partial}{\partial p^{\lambda}}\left[D^{\lambda} \mathcal{F}_{\mu}^{\nu}, N\right]+\frac{1}{2} \frac{\partial}{\partial p^{\nu}} \frac{\partial}{\partial p^{\lambda}}\left[\tilde{F}_{\mu}^{\nu} \tilde{F}^{\mu \lambda}, N\right]\right)+\ldots=0
\end{aligned}
$$

Restriction to the first two terms means the pure classical limit of the mean field (Hartree-) approach (2a) of the equation of motion of matter coupled to a Yang-Mills field. In the Abelian special case of electrodynamics, where $\frac{1}{2}\{\ldots, .$.$\} is replaced by$ the simple product, and the covariant tensor derivative $D_{\mu}(10)$ reduces to the ordinary derivative $d_{\mu}$, this limit is the standard relativistic Vlasov equation [7]. Beyond this limit, to (31) are included field-gradient corrections together with similar contributions of the same order $\hbar$, which, involving commutators, only occur in non-Abelian applications. The standard Vlasov equation appears thus as the Abelian special case of all five terms of the approximation (31) for the general Yang-Mills case.

For a non-Abelian situation eq. (31) can be further reduced by separating the tensor equation as well as its tensor solution

into the trace and the traceless part. With restriction to chromodynamics, one thus obtains the following system coupling the singlet part $n_{0}$ and the octet part $N_{\delta}$ of the wigner transformed density matrix of quark matter

$$
\begin{aligned}
& n_{0} \equiv \operatorname{Tr}, \quad N_{\delta} \equiv N-\frac{1}{3} n_{0} Y: \\
& p^{\mu} \partial_{\mu} n_{0}+p^{\mu} \frac{\partial}{\partial_{p}^{\nu}}\left(T_{r} \mathcal{F}_{\mu}^{\nu} N_{\delta}\right)=0 \\
& p^{\mu} D_{\mu} N_{g}+p^{\mu} \frac{\partial}{\partial p^{\nu}}\left(\frac{1}{2}\left\{\mathcal{F}_{\mu}{ }^{\nu}, N_{g}\right\}-\frac{1}{3}\left(T_{r} \mathcal{F}_{\mu}{ }^{\nu} \mathcal{N}_{8}\right) \mathcal{J}+\frac{1}{3} \mathcal{F}_{\mu}{ }^{\nu} n_{0}\right) \\
& -\frac{i}{8}\left(\frac{\partial}{\partial p^{\nu}}\left[\mathcal{F}^{\mu \nu}, D_{\mu} N_{g}\right]-p^{\mu} \frac{\partial}{\partial p^{\nu}} \frac{\partial}{\partial p^{\lambda}}\left[D^{\lambda} \mathcal{F}_{\mu}^{\nu}, N_{g}\right]+\frac{1}{2} \frac{\partial}{\partial p^{\nu}} \frac{d}{\partial p^{\lambda}}\left[\mathcal{F}_{\mu}^{\nu} \mathcal{F}^{\mu \lambda}, N_{g}\right]\right)=0
\end{aligned}
$$

In a similar way, by adding, instead of subtracting, the contributions of $(29 a, b)$, one obtains a semiclassical expansion of the Hermitian part of eq. (2) 


$$
\left(p^{2}+m^{2}-\frac{1}{4} D_{\mu} D^{\mu}\right) N+\frac{i}{4} p^{\mu} \frac{\partial}{\partial p^{\nu}}\left[F_{\mu}^{\nu}, N\right]+\ldots=0
$$

where, to the order of the commutator terms of (31), corresponding anti-commutators occur plus a product term $F N F$. In the pure classical limit, this equation would read $\left(\rho^{2}+m^{2}\right) N=0$ and just impose the on-shell condition $N \sim \delta\left(p^{2}+m^{2}\right)$ to the solution of the vlasov equation; so (35) may be regarded as some quantum-mechanical modification of this supplementary condition to the general solution of (31).

To close the model of quark-gluon interaction, the generalized VIasov equation describing the motion of guark-matter coupled to a classical gluon field must be completed by a generalized Maxwell equation, which determines this field as the instantaneous mean gluon-field generated by the quark-matter. The mean gluon field may be defined by replacing in the Maxwell-type equation of the quantum field theory the current density operator by its expectation value with regard to the actual quantum-state of the matter

$$
\left.\gamma_{\nu, c^{\prime} c^{\prime \prime}}(x) \equiv \frac{1}{2 i}\left\langle\left(\psi_{c^{\prime \prime}}^{\dagger}(x)_{1}^{\prime} D_{\nu} \psi(x)\right)_{c^{\prime}}-\left(D_{\nu} \psi(x)\right)_{c^{\prime \prime}}^{+} \psi_{c^{\prime}}(x)\right)\right\rangle \text {. }
$$

Due to the octet character of the gluons, the Maxwell equation only involves the traceless part $y_{8}$ of (36)

$$
D_{\mu} \mathcal{F}_{\nu}^{\mu}=g \gamma_{(8) \nu} .
$$

The current density defined by (36) is just given as the first $p$-moment of the solution of the vlasov equation, and the same is true for the traceless parts

$$
\gamma_{(s) \nu}(x)=\int \frac{d^{4} p}{(2 \pi)^{4}} p_{\nu} N_{(g)}(x, p)
$$

this relation is easily proved by insertion of the wigner transform (4), transition to $i^{-4} d / d \xi^{\nu}$ and use of eq. (12) for the $U$ matrix. The covariant continuity equation for $j$ or, as consistent with (36), for $\gamma_{8}$, 
$D_{\mu} \gamma_{(8)}^{\mu}=0$

is obtained by $p$-integration of the vlasov equation.

The semiclassical model developped in this work and given by the coupled equations (33), (34), (37) and (38), together with a supplementary condition of the type (35), may be of use to describe nuclear matter under extreme conditions beyond the deconfinement transition, in particular involving a gluon field sufficiently strong to justify neglection of its quantisation. Apart from inclusion of matter spin, and further quantum corrections of the mattex density matrix which in principle are already provided for in this work, a consequent extension of the model, on the way to describe the deconfinement transition itself, whould also include corrections of the mean field concept due to the quantisation of the gluon field, such as the fluctuations on the background of the mean field. On the other hand, with the aim of some application to astrophysics, one would have to add the coupling to the mean gravity field of the system by a generalization of the formalism for curved space.

\section{References:}

1. see e. g. Satz, H., On critical phenomena in strong interaction physics, Proc. 17th winter School of Theoretical Physics Karpacz (Poland), 1980

2. Kadanoff, L. P. and Baym, G., Quantum Statistical Mechanics, Benjamin, New York 1962

Weigel, M. K. and winter, J., J. Phys. G 9913 (1983)

3. Becher, P., Bohm, M., and Joos, H., Eichtheorien der starken und elektroschwachen Wechselwirkung, Teubner, stuttgart, 1981, p. 97

4. Feynman, R. P., Phys. Rev. 84, 108 (1951)

5. Weinberg, S., Gravitation and Cosmology, Wiley, New York, 1972

6. see.e, g. Ring, P., and Schuck, P., The Nuclear Many-Body

Problem, Springer, Berlin, 1980, p. 535 
7. Stewart, J. M., Non-Equilibrium Relativistic Kinetic Theory, Springer, Berlin, 1971

Ehlers, J., in Proc. Varenna summer School on Relativistic Astrophysics 1969, Sachs, R. K. (ed.), Academic Press, New York, 1971 